\title{
Successfully Pregnancy Outcome After LLETZ in Women with Cervical Intraepithelial Neoplasia: A Case Series
}

\author{
Junita Indarti ${ }^{1 *}$ D, Raymond Surya ${ }^{1}$, Reyhan Aditya ${ }^{1}$, Muhammad Ikhsan ${ }^{1}$, Kristian Alda ${ }^{2}$, Mohammad Agassi Antoniman ${ }^{2}$ \\ ${ }^{1}$ Department of Obstetrics and Gynecology, Faculty of Medicine, Universitas Indonesia, Dr. Cipto Mangunkusumo Hospital, \\ Jakarta, Indonesia; ${ }^{2}$ Faculty of Medicine, Universitas Indonesia, Jakarta, Indonesia
}

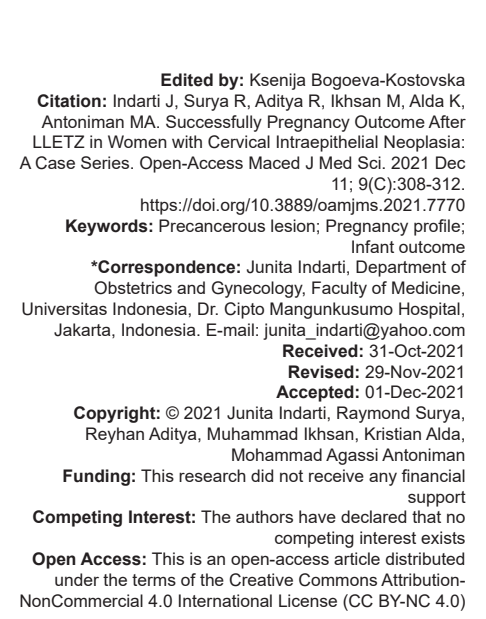

\section{Introduction}

Human papillomavirus (HPV) is a sexually transmitted infection that has an important role in cervical cancer development [1], [2]. The highest risk for HPV infection is in women between 20 and 35 years of age; however, it may occur in younger or older women [3], [4]. The incidence of progressive HPV infection, which is associated with the development of $\mathrm{CIN}$, is highest in 20-25 years old women [5].

The incidence of CIN has been reported from 1.3 to $2.7 / 1000$ pregnancies [6], [7]. The progression rate to cervical cancer of women with CIN 2 and CIN 3 was 2-5 times higher than normal cervix [8]. The previous studies showed that the possibility of regression among pregnant women on CIN 1 was between $32 \%$ and $69 \%$; CIN 2-3 were between $16.7 \%$ and almost $70 \%$ [5]. Based on a study by Kose and Naki [9] about untreated CIN lesions, CIN 1 regression, persistence, and progression rates were $60 \%, 40 \%$, and $10 \%$, respectively; however, for CIN 2, these were $40 \%, 40 \%$, and $20 \%$, respectively. The prognosis of CIN 3 to be invasive cancer was more than $12 \%$. Therefore, cervical cancer screening program is very important and associated with marked reductions in cervical cancer incidence [10].
Some evidence showed that there is a relationship between excisional treatments for CIN such as loop electrosurgical excisional procedure (LEEP) or large loop electrosurgical excisional procedure (LLETZ) and poor obstetric outcomes including low birth weight and preterm birth [6]. The HPV itself may have an association with preterm premature rupture of membranes (PPROM) and other adverse pregnancy outcomes [11].

This case series would like to present women who had been successfully pregnant after treatment of $\mathrm{CIN}$ and associated outcomes also surveillance after it.

\section{Case series}

Patient 1

A 31-year-old P2A0 woman had been performed LLETZ in 2016 due to $A S C H$ results on liquid-based cytology (LBC) and positive high-risk HPV (type 66/88). The histopathology result confirmed CIN I. She underwent annual cotesting with a result of negative LBC and high-risk HPV. Three years after LLETZ, she got pregnant in 2019 and at the age of 31 years old delivered a term baby spontaneously with a birth weight of $3500 \mathrm{~g}$. She had hormonal injection 
contraception as a family planning method. One year later, she did not have any complaints such as postcoital bleeding or vaginal discharge and she underwent an LBC test with a negative result. She was planning for cotesting in 3 years.

\section{Patient 2}

A 34-year-old P2A0 woman had been performed LLETZ in 2017 due to HSIL result on LBC, negative high-risk HPV, and abnormal colposcopy (high-grade lesion). The histopathology result from LLETZ confirmed CIN 2. A year later in 2018, she was performed normal colposcopy with negative LBC result. Then, at the end of 2018, she got pregnant and in the following year at the age of 33 years old, delivered a term baby by cesarean section due to preeclampsia. The birth weight was $3100 \mathrm{~g}$. She had a condom as a family planning method. Annually, she did not have any complaints such as post-coital bleeding or vaginal discharge and she underperformed normal colposcopy and negative LBC results. She was planning for cotesting in 12 months.

\section{Patient 3}

A 35-year-old P3A1 woman had been performed LLETZ in 2017 due to ASCUS results on LBC and positive high-risk HPV (type 52). The histopathology result confirmed CIN 3. After that, she was followed up with negative LBC, negative highrisk HPV. Two years later in 2019, she got pregnant and at the age of 34 years old delivered a term baby by cesarean section due to a previous C-section, not in labor. The birth weight was $3000 \mathrm{~g}$. She had condoms as a family planning method. Annually, she complained vaginal discharge with abnormal colposcopy (low-grade lesion), ASCUS on LBC result, and negative high-risk HPV. She was planned for cotesting in 1 year.

\section{Patient 4}

A 38-year-old P4A0 woman had been performed LLETZ in 2016 due to HSIL result on LBC and positive high-risk HPV (type 33). The histopathology result confirmed CIN 3. After that, she was followed up with normal colposcopy, negative LBC, negative high-risk HPV. One year later in 2018 after LLETZ, she got pregnant and at the age of 36 years old delivered a term baby by cesarean section due to PPROM on 34 weeks and oligohydramnios. The birth weight was $2200 \mathrm{~g}$. She had sterilization as a family planning method. After that, she did not have any complaints of vaginal discharge or bleeding. One year after, she was follow-up with normal LBC and negative high-risk HPV. She was planning for cotesting in 3 years.

\section{Discussion}

In our case series, four women with one ASCUS, one ASCH, and two HSIL were reported. One patient had been performed LLETZ due to ASCUS, one patient due to $\mathrm{ASCH}$ result, and two patients due of HSIL based on LBC; with histopathology result from ASCUS patients were CIN 3, patient with $\mathrm{ASCH}$ was CIN 1, and two patients with HSIL showed a result of CIN 3. The study by Jin et al. [12] showed that LEEP has an association with the risk elevation of preterm birth, PPROM, and low birth weight. They also found that LEEP did not have a significant association with increased risk of cesarean section, perinatal mortality, stillbirth, and neonatal mortality, neonatal intensive care unit admission, and labor induction. In our cases, all of them got pregnant and have good pregnancy outcomes. They got pregnant in one until 3 years after going through excision procedure or colposcopy only. Three pregnancies delivered a term baby and another one was preterm due to PPROM. Three patients delivered the baby by cesarean section due to obstetric indication without regarding the status of precancerous cervical lesion.

From four patients, the age distribution of the patient during pregnancy and delivery varied between 31 and 38 years old. Moreover, in this case, series all patients were having multiparity status, ranging from parity two to parity four. Therefore, education of family planning programs should be incorporated into the management to apply a holistic approach for women with a precancerous cervical lesion in reproductive age. In this case series, following delivery of baby, one patient underwent sterilization, two patients used barrier contraception, and one patient used injection hormonal contraception.

Even though the patients in this case series had advanced maternal age that was associated with adverse pregnancy outcomes, yet only one patient had sterilization that had a $0.5 \%$ unintended pregnancy rate while none other used long-term contraception method. Instead, the other one patient used injection hormonal contraception with a $6 \%$ unintended pregnancy rate, and the other two patients used the condom method with an $18 \%$ unintended pregnancy rate [13]. Studies had shown that advancing maternal age was associated with miscarriage, chromosomal abnormalities, fetal growth restriction, preeclampsia, and gestational diabetes. Advancing maternal age is a strong independent risk factor for miscarriage with the lowest risk of first-trimester loss of $8-10 \%$ at the age of twenties that undergo steep rise from the age of 30 . The rate of first-trimester loss reached $17-25 \%$ in women aged 35-40 years old. Furthermore, the risk of chromosomal abnormality increased exponentially from the age of 35 to 39 years old reaching $0.8-1.3 \%$. The same applied to the incidence of fetal growth restriction that has increased odds ratio of 1.2-1.6 from 
the age of 35 to 40 years old. Maternal complications also increased with increasing age because the risk for preeclampsia and gestational diabetes mellitus increased continuously [14]. Therefore, it was important for the patients in this case series with advanced maternal age and no desire for future fertility to opt for a long-term or permanent method of contraception for safe motherhood during visits for follow-up of the cervical precancerous lesion.

Based on a retrospective study by $\mathrm{He}$ et al. [8], the rate of cesarean section was associated with CIN grades that elevation of CIN grades would increase the prevalence of cesarean section rate. Of 88 pregnant women, $56(63.6 \%)$ patients have performed a cesarean section. They also explained that there was no significant difference between pregnant women with $\mathrm{CIN}$ or cervicitis and the occurrence of PROM, polyhydramnios, cervical laceration and postpartum hemorrhage, placental abruption, low birth weight, neonatal deformity, and asphyxia. On the other side, there was higher premature infants and oligohydramnios incidence in pregnant women with ASCUS than in the cervicitis group [8].

Kalliala et al. [15] presented the first pregnancy and live birth incidence was significantly increased after $\mathrm{CIN}$ treatment. There was no increase in the incidence of pregnancy termination, extrauterine pregnancy, miscarriage, and molar pregnancy after the treatment. They also commented that CIN treatment did not decrease the incidence of pregnancy and more pregnant women had a live birth after treatment [15]. However, in a study by Kyrigou et al. [4], the pregnancy rate after CIN treatment was presented. There was a higher rate of pregnancy for treated women than untreated women. In our study 1-3 years after treatment, the patient got pregnant. There were similar rates between treated and untreated women in total and first-trimester miscarriage, but increased second-trimester miscarriage risk, ectopic pregnancy, and termination were associated with the CIN treatment [4]. Therefore, in our cases, there was a normal phenomenon that our patients of reproductive ages got pregnant after CIN treatment.

Pandey et al. [3] and Nimrodi et al. [16] study concluded that there is no association between HPV type and pregnancy complications such as PROM, PPROM, preterm birth, preeclampsia, placental abruption, and cervical insufficiency. It is hypothesized that the biological behavior of high-risk HPV in pregnant women was less aggressive compared with non-pregnant women. This study evaluated Ki-67 and p16 immunostaining, which can be useful indicators of HPV infections and lesion severity, especially for HR-HPV. Ki-67 and p16 had less expression in pregnant women and these results suggest that pregnancy can exhibit cellular protein expression that has a role in the carcinogenic process [17]. Different from both studies above, the meta-analysis study by Niyibizi et al. [18] found that HPV was associated with preterm birth and also PPROM significantly. They also said that
HPV may also relate to fetal death, IUGR, and low birth weight. The study by Hong et al. [19] also found that there was no significant difference between pregnant women's abnormal LBC or HR-HPV-positive and normal pregnant women. They also commented that HPV positive may be associated with preterm birth. Meta-analysis suggested that pregnant women previously treated by LLETZ are at approximately twice the risk of preterm birth than pregnant women, in general [20]. In our case series, one of the patients was delivered the baby prematurely due to PPROM.

The 2019 ASCCP guidelines are the guidelines for cervical cancer screening abnormalities management and these guidelines use equal management for equal risk principle. The risk calculation is based on the risk of the patient developing cervical cancer and 5-year risk of CIN progression [21], [22]. Perkins et al. [21] also announced that the more frequent surveillance and treatment are highly recommended for higher progressive risk patients. On the other side, the lower risk patient can wait for colposcopy and do longer intervals of follow-up, but still, need a return to routine screening [21]. The management of precancerous cervical based on risk is shown in Figure 1 [22].

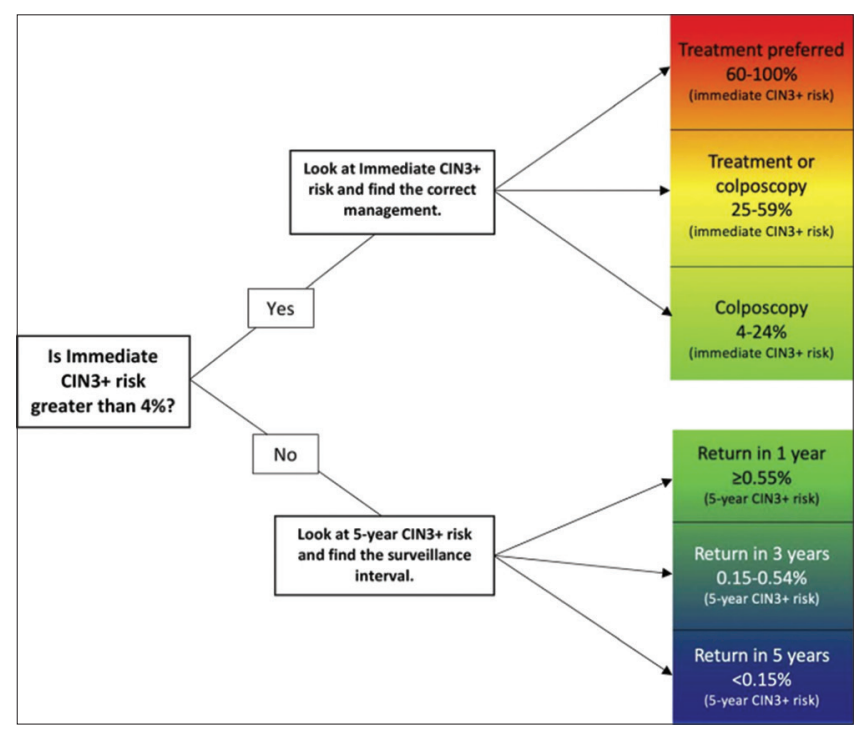

Figure 1: Determining suggested management based on calculated CIN3+ risk [19]

The study by Pitner et al. [1] explained that high regression rates of colposcopy and LBC result after delivery. By 8 weeks after delivery, regression of the disease with Pap smear showed $63 \%$ and $53 \%$ normal LBC result, only $3 \%$ had progression. On the other sides, 15 patients have performed a colposcopy-guided biopsy and showed $67 \%$ HSIL, $13 \%$ LSIL, and $7 \%$ invasive carcinoma 8 weeks after delivery [1].

A faster treatment is preferred for 25 years or older non-pregnant patients with HSIL and concurrent positive testing for HPV genotype 16 (HPV 16) and the patients with HPV-positive HSIL regardless of HPV genotype that never or rarely screened [21]. 
Surveillance with HPV testing or cotesting at 3-year intervals for a minimum of 25 years is recommended after treatment and initial post-treatment management of histologic HSIL, CIN 2, CIN 3, or AIS. However, it is still acceptable with consideration of the patient's life expectancy of Perkins et al. [21].

Surveillance with only cytology is acceptable if HPV testing or cotesting is not available. Cytology is less sensitive than HPV testing for pre-cancer detection and is recommended more often. When HPV testing or cotesting is recommended annually, cytology is recommended at 6-month intervals. However, when HPV or cotesting is recommended for 3-year intervals, the annual cytology is the recommendation [21]. On our cases, annually after delivery, these patients had been observed by LBC and/or colposcopy. It should be conducted by cotesting in 3 years, except case with $\mathrm{CIN}$ 2 on histopathological without data of annual follow-up with cotesting in 12 months.

For the first, second, and fourth patients, which had negative LBC tests, cotesting should be conducted 12 months or 3 years based on screening status. HPV testing is preferred due to its sensitivity. Routine screening with cytology is performed every 3 years if the cytology result is negative for the second cotesting. If cytology result is more than ASC or HPV testing is positive, the patients should be performed colposcopy. The third patient, which had an ASCUS LBC result, had been performed colposcopy and got a normal result of colposcopy. HPV testing is preferred to see the risk of this patient. Cotesting is should be done for 3 years regarding negative high-risk HPV. Therefore, the patient had an individualized algorithm based on CIN status on histopathological results and the management should follow ASCCP guidelines.

\section{Conclusion}

The cervical precancerous lesion does not decrease the rate of pregnancy; however, treatment of CIN will increase the pregnancy rate and it is not associated with obstetric complications. Although pregnancy will delay the surveillance of precancerous lesions, it is recommended to follow ASCCP guidelines to decrease the risk of progressing to cervical cancer.

\section{References}

1. Pitner I, Orsag ND, Škrtić B, Čukelj M, Kuna K, Tučkar N, et al. Colposcopy significance in cervical premalignant lesions during pregnancy. J Gynecol Surg. 2017;33(5):189-92.
2. deSanjoseS, QuintWG,AlemanyL, GeraetsDT,KlaustermeierJE, Lloveras B, et al. Human papillomavirus genotype attribution in invasive cervical cancer: A retrospective cross-sectional worldwide study. Lancet Oncol. 2010;11(11):1048-56. https:// doi.org/10.1016/S1470-2045(10)70230-8

\section{PMid:20952254}

3. Pandey D, Solleti V, Jain G, Das A, Prasada KS, Acharya S, et al. Human papillomavirus (HPV) infection in early pregnancy: Prevalence and implications. Infect Dis Obstet Gynecol. 2019;2019:4376902. https://doi.org/10.1155/2019/4376902 PMid:31019362

4. Kyrgiou M, Mitra A, Arbyn M, Paraskevaidi M, Athanasiou A, Martin-Hirsch PP, et al. Fertility and early pregnancy outcomes after conservative treatment for cervical intraepithelial neoplasia. Cochrane Database Syst Rev. 2015;2015(9):CD008478. https:// doi.org/10.1002/14651858.CD008478.pub2

PMid:26417855

5. Mailath-Pokorny M, Schwameis R, Grimm C, Reinthaller A, Polterauer S. Natural history of cervical intraepithelial neoplasia in pregnancy: Postpartum histopathologic outcome and review of the literature. BMC Pregnancy Childbirth. 2016;16(1):74. https://doi.org/10.1186/s12884-016-0861-8

PMid:27055819

6. Aitken CA, Siebers AG, Matthijsse SM, Jansen EE, Bekkers RL Becker $\mathrm{JH}$, et al. Management and treatment of cervical intraepithelial neoplasia in the Netherlands after referral for colposcopy. Acta Obstet Gynecol Scand. 2019;98(6):737-46. https://doi.org/10.1111/aogs.13547

PMid:30687935

7. Han SN, Mhallem Gziri M, Van Calsteren K, Amant F. Cervical cancer in pregnant women: Treat, wait or interrupt? Assessment of current clinical guidelines, innovations, and controversies. Ther Adv Med Oncol. 2013;5(4):211-9. https:// doi.org/10.1177/1758834013494988

PMid:23858330

8. He Y, Wu YM, Wang T, Song F, Wang Y, Zhu L. Perinatal outcomes of pregnant women with cervical intraepithelial neoplasia. Arch Gynecol Obstet. 2013;288(6):1237-42. https:// doi.org/10.1007/s00404-013-2889-6

PMid:23695508

9. Kose FM, Naki MM. Cervical premalignant lesions and their management. J Turk Ger Gynecol Assoc. 2014;15(2):109-21. https://doi.org/10.5152/jtgga.2014.29795 PMid:24976778

10. Practice Bulletin No. 168: Cervical cancer screening and prevention. Obstet Gynecol. 2016;128(4):e111-30. https://doi. org/10.1097/AOG.0000000000001708 PMid:27661651

11. Goulding AN, Rahangdale L. Human papillomavirus and adverse pregnancy outcomes: An opportunity for prevention? J Womens Health (Larchmt). 2019;28(5):565-7. https://doi. org/10.1089/jwh.2018.7602

PMid:30681388

12. Jin G, LanLan Z, Li C, Dan Z. Pregnancy outcome following loop electrosurgical excision procedure (LEEP) a systematic review and meta-analysis. Arch Gynecol Obstet. 2014;289(1):85-99. https://doi.org/10.1007/s00404-013-2955-0

PMid:23843155

13. Curtis KM, Tepper NK, Jatlaoui TC, Berry-Bibee E, Horton LG, Zapata LB, et al. Medical eligibility criteria for contraceptive use. MMWR Recomm Rep. 2016;65(3):1-103. https://doi. org/10.15585/mmwr.rr6503a1 PMid:27467196

14. Frick AP. Advanced maternal age and adverse pregnancy outcomes. Best Pract Res Clin Obstet Gynaecol. 
2021;70:92-100. https://doi.org/10.1016/j.bpobgyn.2020.07.005 PMid:32741623

15. Kalliala I, Anttila A, Nieminen P, Halttunen M, Dyba T. Pregnancy incidence and outcome before and after cervical intraepithelial neoplasia: A retrospective cohort study. Cancer Med. 2014;3(6):1512-6. https://doi.org/10.1002/cam4.300 PMid:25146172

16. Nimrodi M, Kleitman V, Wainstock T, Gemer O, Meirovitz M, Maymon $\mathrm{E}$, et al. The association between cervical inflammation and histologic evidence of HPV in PAP smears and adverse pregnancy outcome in low-risk population. Eur J Obstet Gynecol Reprod Biol. 2018;225:160-5. https://doi.org/10.1016/j. ejogrb.2018.04.023

PMid:29727786

17. Ciavattini A, Sopracordevole F, di Giuseppe J, Moriconi L, Lucarini G, Mancioli F, et al. Cervical intraepithelial neoplasia in pregnancy: Interference of pregnancy status with p16 and Ki-67 protein expression. Oncol Lett. 2017;13(1):301-6. https://doi. org/10.3892/ol.2016.5441

PMid:28123559

18. Niyibizi J, Zanré N, Mayrand MH, Trottier H. Association between maternal human papillomavirus infection and adverse pregnancy outcomes: Systematic review and meta-analysis. J Infect Dis. 2020;221(12):1925-37. https://doi.org/10.1093/infdis/jiaa054

PMid:32022858
19. Hong JN, Berggren EK, Campbell SL, Smith JS, Rahangdale L. Abnormal cervical cancer screening in pregnancy and preterm delivery: Abnormal cervical screening and preterm delivery. Paediatr Perinat Epidemiol. 2014;28(4):297-301. https://doi. org/10.1111/ppe.12132

PMid:24891016

20. Sasieni $\mathrm{P}$, Castanon A, Landy R, Kyrgiou M, Kitchener $\mathrm{H}$, Quigley $\mathrm{M}$, et al. Risk of preterm birth following surgical treatment for cervical disease: Executive summary of a recent symposium. BJOG. 2016;123(9):1426-9. https://doi. org/10.1111/1471-0528.13839

PMid:26695087

21. Perkins RB, Guido RS, Castle PE, Chelmow D, Einstein MH, Garcia F, et al. 2019 ASCCP risk-based management consensus guidelines for abnormal cervical cancer screening tests and cancer precursors. J Low Genit Tract Dis. 2020;24(2):102-31. https://doi.org/10.1097/ LGT.0000000000000525

PMid:32243307

22. Egemen D, Cheung LC, Chen $X$, Demarco $M$, Perkins RB, Kinney W, et al. Risk estimates supporting the 2019 ASCCP risk-based management consensus guidelines. J Low Genit Tract Dis. 2020;24(2):132-43. https://doi.org/10.1097/ LGT.0000000000000529

PMid:32243308 